\title{
Traitements sémantiques du lexique français (1550-1694). Vers l'invention du dictionnaire monolingue
}

\author{
Simone Delesalle \\ Université Paris VIII - Laboratoire HTL Paris VII - CNRS \\ rowlson.delesalle@,noos.fr \\ Francine Maziere \\ Université Paris XIII - Laboratoire HTL Paris VII - CNRS \\ frmaziere@wanadoo.fr
}

Le «mot français » est une construction. Son traitement s'élabore sur plus d'un siècle avant les premiers dictionnaires monoligues (1550-1680). En 1550, Meigret déclare qu'il «faut une bonne oreille » pour reconnaître un mot français dérivé du latin oral. Il lui reconnait essentiellement un phonétisme et une morphologie. Le mot français est constitué «étymologiquement», au sens classique du terme, à savoir par son origine (latin, grec, hébreu...) et par sa dérivation. Estienne reprend le terme dans sa grammaire et ses dictionnaires bilingues latin-français et français-latin.

Cent ans plus tard paraissent des outils attendus: des dictionnaires monolingues, Richelet en 1680, Furetière en 1690, le Dictionnaire de l'Académie en 1694 (désormais DA), qui abandonnent toute référence à l'origine. Les deux premiers, parce qu'ils appliquent l'ordre alphabétique, négligent la morphologie. Seul le DA fonctionne comme un dictionnaire raisonné du lexique : il classe les dérivés sous une même entrée (humainement sous homme et néanmoins sous ne), recourt à la définition morphologique selon les parties du discours, et il inclut aussi dans son traitement ce qu'inventèrent certains petits traités intermédiaires : la collocation (travail syntagmatique) et la différenciation des sens (travail en paradigme et sur les emplois), ce qu' au XVIII ${ }^{\mathrm{e}}$ siècle on appellera la « justesse » du mot.

C'est ce cheminement que nous nous proposons d'illustrer en nous appuyant sur les travaux des dix dernières années en histoire des outils linguistiques, travaux qui ont (1) redonné sa place au Dictionnaire de l'Académie, dictionnaire de langue et non de chose ou de rhétorique, inventeur d'une "langue commune » en sa première édition (1686-1694); (2) montré l'apport, par principes et technicité, des traitements spécifiques du lexique hors dictionnaires.

Nous essaierons ici de mettre en évidence la diversité des traitements de sémantique lexicale et leur stabilisation. Ceci nous conduira à bousculer la doxa sur une histoire continue des dictionnaires (Estienne, Nicot, Richelet) où le français gagnerait naturellement du terrain, au profit d'un examen des apports décisifs d'outils composites : grammaires incluant des listes de mots, voire des dictionnaires restreints, remarques et groupements raisonnés de remarques, méthodes pédagogiques, traitements de propriétés particulières comme la synonymie.

Dans un premier temps, nous rappellerons les difficultés de constituer un lexique français, à la Renaissance, puisque cela revient à le définir dans cet ensemble dynamique qui constitue une « hyperlangue » (Auroux, 1998, 2007). En l'occurrence, un co-linguisme latin-français. Nous verrons dans un deuxième temps, beaucoup plus développé, comment ces unités de sens sont traitées dans divers outils linguistiques. 


\title{
1.- Reconnaître le français comme langue
}

\subsection{En grammaire}

Pour reconnaître le français comme langue, il a fallu l'autonomiser à partir de la «séparation » du français opérée au $\mathrm{XVI}^{\mathrm{e}}$ siècle. C'est ce souci d'autonomisation qui caractérise l'attitude du premier grammairien qui écrive, en 1550, une grammaire du français en français, Louis Meigret. (Les précédentes étaient en effet écrites en latin, beaucoup des suivantes le seront aussi). Reconnaissant que le français dit le sens par des mots hérités, Meigret tente de donner des règles de transformation morphologique et de cerner ce qui, de l'héritage, est chassé par l'adoption d'un culturel spécifique. Dans le multiple empirique qu'il traite, il y a bien héritage :

Les François ont fait leurs mots des Hébreux, des Grecs et des Latins ${ }^{1}$.

Il y repère des adaptations morphologiques :

Les François font leurs noms latins en $o$ en y ajoutant $n$ à la façon des Grecs à moins que tu ne préfères que cela se fasse par apocope à partir du génitif comme...

Il repère également des variations diatopiques et/ou distratiques reliées elles-mêmes à l'héritage, portant sur les composants phoniques et graphiques des vocables :

\begin{abstract}
[40-19] Il est vrai, toutefois, qu'il est bien raisonnable que quand quelque vocable est diversement prononcé et avec quelque débat, de sorte que l'autorité de l'usage branle, de remettre sus celui qui a la meilleure apparence. Celui me semble l'avoir meilleure qui a quelque support de règle : comme par exemple ce terme liveao, lequel les maçons de Paris ont corrompu avec son dérivé liveler, disant niveao, niveler : quoiqu'assez de maçons d'autres contrées de France et les hommes entendus en notre langue ne les ensuivent pas. A bonne raison, donc, nous dirons liveao et liveler: vu qu'ils sont descendus de livre signifiant un certain poids : et que les dictions dont ils sont tirés sont libella et librar.
\end{abstract}

Avec un souci marqué pour les variations de prononciation pour une même graphie :

\begin{abstract}
Je vous laisse à penser quelle grâce aura l'e clos en ces vocables mes, tes, ses, si nous l'y prononçons comme nous faisons en pere, mere : et comme font je ne sais quels efféminés mignons avec un presque clos resserrement de bouche : craignant à mon avis que la voix virile de l'homme ne soit point tant harmonieuse ni agréable aux dames qu'une lâche, faible et féminine. Or, quant à moi, je ne poursuis pas ici cette douillette et efféminée façon de parler : car je la laisse aux amoureux, poursuivant tant seulement cette générale et commune façon qui sent son homme et qui est reçue entre les mieux appris. Or, que l' $e$ ouvert ne puisse être prononcé pour l' $e$ clos, cette niaise prononciation que font aucuns des Parisiens (comme je vous l'ai dit autrefois) en la dernière syllabe des secondes personnes du pluriel du futur de l'indicatif et en la même du présent de l'optatif nous en donne une potable connaissance, quand ils prononcent donores, doneries : pour donerez, doneriez.
\end{abstract}

Mais Meigret souligne aussi des idiosyncrasies logées au cœur d'ensembles lexicaux :

\begin{abstract}
Au regard des autres espèces des noms propres, que les Latins appellent pronomen, nomen, cognomen et agnomen, les Français communément ne gardent que le nom et le cognomen que nous appelons le surnom : parce que c'est le nom commun à toute la race [c'est notre «nom de famille»]. Quelquefois aussi nous usurpons quasi comme pour une grande gloire les noms des seigneuries, métairies, moulins, buissons, montagnes, vallées, prés, haies, chaussées : finalement, il semble que le Français fait si peu de compte de porter le surnom de sa race qu'en le délaissant, il s'usurpe le nom de ses possessions et seigneuries : et en défaut d'elles, il s'en forge sur des buissons, haies, loups et renards : comme Lovetiere, Renardiere, Bruyere
\end{abstract}

\subsection{Du côté des dictionnaires}

Ils sont nombreux au XVI ${ }^{\mathrm{e}}$ siècle : environ 150 entre 1540 et 1600, en comptant les rééditions, toujours biou plurilingues (Calepino va jusqu'à 11 langues), et toujours, s'ils sont monolingues, listant des termes 
spécialisés ou des «phrases », des «manières de parler». Ils s’intitulent souvent « inventaire », « magasin », « porte ». Les dictionnaires d'Estienne, latin-français puis français-latin, plus complets, restent isolés et affichent clairement le latin. Le Dictionnaire de Nicot, qui le reprend puis le renouvelle, souvent cité, à juste titre, comme introduisant des amorces de définitions en français, reste dépendant de l'équivalence latine :

- soit par une simple série :

Bastir, Architectari, Condere, AEdificare, Exaedificare, Struere, Praestruere, Instaurare.

- soit par la mise en œuvre d'une série de mots latins à travers des expressions, sans distinction des divers sens en français :

Terre, f. penac. Est l'un des ouvrages premiers de Dieu, attribué aux hommes, où tous animaux et alimens d'iceux sont placez, et est le centre du comble de l'air.

Terra, dont il est parti : l'Italien suit le Latin, disant terra, l'Espagnol l'admignote disant tierra. Tellus, Humus, Solum.

Terre franche, est celle qui n'est point entremeslée de gravier, tuf, ne de quantité de pierres addentées, Pura terra, mera Tellus. Et est l'opposite de Tuf, voyez Tuf.

Naviger et aller terre à terre, Ad oram, Ad littus nauigare.

Aller habiter en terre estrange, Mutare solum.

Terre forte et grasse, Densa et glutinosa terra.

Terre grasse et ferme, Spissa humus. Terre fertille et bien grasse, Grauis terra, Solum laetum. [... $]^{2}$

Nicot était encore dans l'origine latine, même dans les développements les plus importants en français. Dans l'exemple suivant, la dérivation n'est pas distinguée et n'est donc pas listée. Les dérivés servent à définir :

Couple, f. C'est ce qui se consiste en deux ou de deux, ou pour mieux dire les deux choses mesmes, comme une couple de chevaux, Par equorum. Il vient de Copula, qui vient de Copulare, mettre deux choses de mesme espece ensemble : car on ne dira pas une couple, d'un cheval et d'un boeuf. [...]

Couple aussi est une cordelette à accoupler les chiens pour les mener en lesse de deux en deux : de là viennent ces manieres de parler en fait de venerie coupler ou accoupler et descoupler chiens, Copula, hinc apud Ouidium, Copulam detrahere canibus, Descoupler les chiens.

Aller en couple, c'est aller accouplez, s'entre-tenans de deux en deux. Fouillous au chap. 11. apres que aurez retiré les chiens au chenil, il leur faut pendre des billots de bois au col pour leur apprendre à aller en couple.

\section{$1.3 \quad$ Les traités particuliers}

De fait, la singularité des traitements se manifeste moins dans des ouvrages répertoriés comme grammaires ou dictionnaires que dans des traités où la langue française est « objet » à voir de l'extérieur, objet d'enseignement par exemple, comme dans des manuels à visée d'apprentissage (De Vivre : Synonimes, 1569), ou dans des traités de bien écrire (La Porte : Les Epitètes 1571). Les travaux d'Odile Leclercq analysent cette invention par l'épithète, par l'organisation alphabétique des proverbes, par des lexiques techniques de ce qui deviendra la définition lexicographique. Nous y renvoyons. 


\section{2.- Après 1650}

Richelieu a créé l'Académie française en 1636 pour arrêter une langue claire et non équivoque qui puisse servir à l'administration d'un royaume centralisé. Vaugelas, en charge de la rédaction du Dictionnaire, publie en 1647 des Remarques sur la langue française, premier jalon d'un genre repris et amendé durant plus de 50 ans. Il s'agit d'organiser la mise en règles de la langue à partir de principes et en en « réduisant» (c'est à dire, conformémént aux emplois de l'époque, en en «ordonnant») les variations. Plus que jamais, grammaire et lexique ont partie liée. Cela aboutira à ces « minuties grammaticales » que revendique hautement le DA.

Concourent alors à ordonner le lexique, outre les traités particuliers et les grammaires, les Remarques qui, peu à peu, vont gagner en ordre, jusqu'au Génie de la langue française de d'Aisy, en 1685. On peut suivre ces mises en place d'abord par l'étude d'une propriété, la synonymie, puis par le rôle d'une technicité, la mise en liste, dans des grammaires, enfin par des organisations macrostructurelles dont le dictionnaire de langue monolingue est un exemple accompli.

\section{$2.1 \quad$ La synonymie}

En ce siècle, la synonymie est d'abord traitée dans des ouvrages spécifiques comme celui de Montméran (Synonimes et epithètes françoises, 1645), qui, reprenant explicitement La Porte, procède en deux étapes : la liste des synonymes est donnée en premier, suivie de celle des épithètes :

FAMILIARITÉ ; conversation : fréquentation ; accointance : privauté ; société : compagnie.

Douce, privée, étroite, libre, réciproque, mutuelle, fidèle, secrette, amiable, amoureuse, grande, sociable,

consolatoire, fréquente, domestique, commensale, singulière, ordinaire, indissoluble, fraternelle, commune, inviolable

FAVEUR, grace, courtoisie, bien-fait, bon office, civilité

Extraordinaire, spéciale, singulière, particulière, inusitée, grande, douce, bénigne, gracieuse, courtoise, amiable, secourable, opportune, estimable, mondaine, trompeuse, ondoyante, flottante, fragile, vaine, populaire, ambitieuse, journalière, courtisane, excellente, non-pareille, officieuse.

Pour familiarité nous trouvons alors privauté en synonyme et privée en épithète. Faveur a pour premier synonyme grâce, et peut être gracieuse... La démonstration de cette circulation morpho-sémantique pourrait continuer. On voit qu'elle est circulation du concept dans les parties du discours, et qu'elle n'a rien d'une circularité vaine. Elle est aussi développement des attributs du substantif, somme de ses qualités, donc chemin vers sa définition. Plus précisément, on voit combien l'épithète est porteuse du sens du substantif qui la «connote» pour reprendre une formulation de Port-Royal. Nous renvoyons pour plus de précisions, la encore au travail d'Odile Leclercq (thèse en cours de publication) :

\footnotetext{
Ce jeu morpho-sémantique semble mettre en valeur l'utilisation possible de l'épithète, devenant quasiment épithète définitoire, pour «dire » le sens du nom, et donc l'utilisation possible de l'usage pour expliciter le sens d'un mot.

L'hypothèse que l'épithète, au départ «fait de discours », ait pu jouer un rôle d'outil sémantique au moment de l'élaboration des premiers traitements du lexique français, c'est-à-dire un rôle d'outil servant à représenter le sens des mots, semble confirmée par l'observation de la place accordée par les académiciens à la collocation «nom + adjectif » dans leur dictionnaire, au mépris des nombreuses railleries de Furetière.
}

Les interrogations que l'on trouve dans ces traités spécifiques relèvent d'une quête de l'organisation, garante de stabilisation. Ils sont à la fois des témoignages de l'usage et des modélisations.

Mais l'inventeur des Remarques, Vaugelas, joue dans ce domaine un rôle important, puisque, sans affirmer, comme on le fera à la fin du siècle, principalement dans le DA, l'absence nécessaire de vrai synonyme dans une langue, il se sépare également des pratiques du siècle précédent que Claude Buridant 
a mis en lumière. Celui-ci a montré en effet le «binôme synonymique » du moyen français comme une spécification du sens, soulignant (1), la valeur de «vecteur sémantique » du deuxième synonyme, destiné à restreindre ou à recentrer le sens dans des cas de polysémie (on est fier et orgueilleux face à l'ennemi, qui est fier et féroce) ou à «acclimater» en français un mot latin, savant (vélocité et hastiveté); (2), l'évolution historique de cette pratique et son fort déclin au XVII ${ }^{\mathrm{e}}$ siècle, à partir du moment où la préférence est accordée au «mot juste », ce qui relève d'une position idéologique sur la langue ; et (3), la concentration des emplois dans les traductions et les formulaires, deux lieux d'où il faut bannir l'équivoque, partant, travailler un ordre de la langue où le sens soit arrêté. Vaugelas, lui, fait du second synonyme, coordonné, une accentuation, « un second coup de pinceau qui achève l'image » :

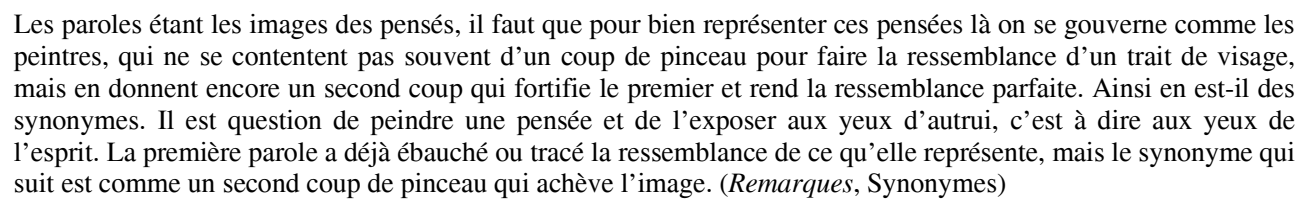
peintres, qui ne se contentent pas souvent d'un coup de pinceau pour faire la ressemblance d'un trait de visage, mais en donnent encore un second coup qui fortifie le premier et rend la ressemblance parfaite. Ainsi en est-il des synonymes. Il est question de peindre une pensée et de l'exposer aux yeux d'autrui, c'est à dire aux yeux de l'esprit. La première parole a déjà ébauché ou tracé la ressemblance de ce qu'elle représente, mais le synonyme qui suit est comme un second coup de pinceau qui achève l'image. (Remarques, Synonymes)

Par ailleurs Vaugelas, dans certaines de ses Remarques, oriente ses analyses vers un autre jeu synonymique, la synonymie distinctive, l'un des mots excluant l'autre. Il ne s'agit plus de la succession discursive mais du choix d'un mot dans la perspective de tel ou tel contexte d'emploi. Par là s'annonce l'ouvrage fondamental de l'abbé Girard via la préface du Dictionnaire de l'Académie (1694) :

[...] les synonymes, c'est-à-dire les mots qui sont de même signification ; sur quoi on croit devoir avertir que le synonyme ne répond pas toujours exactement à la signification du mot dont il est synonyme, et qu'ainsi ils ne doivent pas être employés indifféremment l'un pour l'autre.

Le continuateur immédiat de Vaugelas est Jean Macé, un prédicateur proche de Richelieu. Son travail s'inscrit sans équivoque dans les ouvrages de français à l'usage des étrangers. Il réécrit, simplifie, reprend les Remarques en tentant un classement par ordre alphabétique. Le traitement des doublets et des synonymes, qui sont la trace, dans l'usage, des imperfections contre la raison (l'équivoque), suit en grande partie Vaugelas. Mais Macé le durcit, en accentuant, par la simplification, les restrictions d'emploi («Face est un mot condamné pour dire visage, si ce n’est en matière de dévotion »).

On a là l'amorce d'un discours nouveau, celui des prescriptions en langue. Dans cette nouvelle approche des variations, les indications de domaine («en matière de dévotion ») se retrouveront dans l'organisation du DA qui mettra en place une métalangue sélective et, surtout, la collocation comme environnement linguistique et sociolinguistique de la synonymie. Plus largement, les grammairiens et les remarqueurs travaillent, au milieu du siècle, à réunir, dans leurs études du lexique, morphologie, sémantique et histoire des mots.

\subsection{Les listes dans des grammaires : poursuite d'une exploration raisonnée}

Les ouvrages qui s'écartent des Remarques ponctuelles pour s'organiser en grammaires du français offrent des listes lexicales dans lesquels ont retrouve des réductions de synonymie, mais prises dans des considérations grammaticales : la dérivation et les parties du discours. On retrouvera dans le DA ces deux approches, qui élaborent le plus sûrement les nouvelles possibilités de dire le sens.

Entre 1656 et 1660 paraissent les ouvrages de Claude Irson (Nouvelle Méthode pour apprendre facilement les principes et la pureté de la langue française, 1656 et 1660) et de Chiflet (Essai d'une parfaite grammaire de la langue française, 1659). Ce sont des grammaires parues presque en même temps, qui comportent des développements conséquents sur le lexique français. Mais ces ouvrages ne visent pas le même public. Chiflet se soucie de faire connaître la langue française aux étrangers lettrés (il y a dans le texte une partie consacrée aux lecteurs flamands). Irson, lui, s'adresse en particulier aux gens qui n'ont pas appris le latin et ont peur d'être moqués dans les conversations. Par ailleurs Chiflet est un 
jésuite, tandis que Irson est lié, d'une manière non encore réellement explicitée mais certaine, au groupe de Port-Royal. Leurs options sur la langue s'en ressentent.

Les deux grammairiens sont confrontés, dans une diachronie très courte, à l'examen des variations en référence à l'usage et à la raison.

Chiflet propose des «Observations » qui suivent les chapitres consacrés aux mots lexicaux de la langue, en liste ouverte. Elles se situent donc à la fin de chaque partie du discours. Autrement dit ces listes se situent à l'intérieur d'un système grammatical qui a en charge avant tout de repérer les espèces de mots. On reste là dans le domaine des «modes de signifier ». Ces « observations » sont liées aux analyses contemporaines des Remarqueurs. Les plus nombreuses concernent la manière dont les mots sont reçus, ce qui touche souvent à des points de synonymie, à l'usage du sens figuré, aux archaïsmes et aux néologismes. Parmi les nombreuses références explicites à Vaugelas, on trouve celle-ci, au début des « observations des noms » :

\footnotetext{
Commençons par ceux dont l'usage est contesté entre l'Auteur des Remarques et ceux qui lui ont contredit par leurs Censures. La Remarque rejette Bienfacteur et Malfacteur et substitue en leur place Bienfaiteur, Malfaiteur. [...]. 2- Je mettrai ensuite les noms qui sont condamnés communément de tous. Banquet ne se dit plus que des choses sacrées et Banqueter est hors d'usage. [...].
}

Même si, dans la suite de ses observations, Chiflet traite les noms d'une manière de plus en plus brève et renonce à un ordre raisonné au profit de l'alphabet, on trouve là des renseignements précis et précieux sur l'importation récente de substantifs abstraits tirés du latin classique (insidieux, sécurité), sur le rejet des archaïsmes, et sur la différence entre les formes utilisées à la cour et celles qui sont courantes dans le peuple.

On trouve dans cet ouvrage des analyses intéressantes qui touchent au lien entre forme et sens des mots, ou bien à leur sens seul. D'un côté, la confrontation détaillée entre paronymes ou entre quasi synonymes : chaire et chaise, fureur et furie, nouveau et neuf, etc. De l'autre, la distincion entre synonymes, approchants et contraires :

\footnotetext{
La différence qu'il y a entre nouveau et neuf, c'est que neuf signifie quelque chose fait par art, qui n'est point encore mise en usage. Un livre neuf, qui n'a pas encore été usé ni sali, en le maniant, quoique peut-être il soit imprimé et relié depuis beaucoup d'années. Nouveau est ce qui est fait, ou mis en évidence, depuis peu de temps, comme un nouveau livre, qui a été nouvellement composé, encore qu'on aurait déjà flétri les feuillets et sali la couverture, à force de le lire et de le relire, et qu'à cause de cela on ne saurait plus l'appeler un livre neuf. Vous voyez clairement par cet exemple qu'une chose peut être neuve sans être nouvelle, et nouvelle sans être neuve.
}

D'un autre côté, la distinction entre synonymes, approchants et contraires :

\begin{abstract}
Il y a déjà plusieurs règles touchant la répétition, semées en divers endroits de cette grammaire, aux places qui semblaient les exiger. Je mettrai ici le reste. Mais pour entendre les règles suivantes, il faut présupposer avec M. de Vaugelas qu'il y a des mots qui sont synonymes, d'autres qui sont contraires. Les synonymes sont ceux qui ont une même signification : comme aimer, chérir. A ces synonymes se rapportent les approchants, qui ont presque la même signification, comme aimer, révérer. Les contraires sont ceux qui ont une signification, directement opposée, comme bâtir et démolir. Il faut réduire à ces contraires les différents, qui ont une signification éloignée et différente l'un de l'autre, comme bâtir et fortiifier.
\end{abstract}

Irson a, par rapport à Chiflet, une vision plus autonome du lexique qui dépasse le cadre des parties du discours. On dispose dans ses Méthodes de deux parties lexicales bien distinctes, qui s'insèrent dans la grammaire en passant des modes de signifier à la signification : elles sont constituées d'entrées classées alphabétiquement, et dans lesquelles figurent à la fois des noms, des verbes et des adverbes, ce qui les apparente à des études d'ordre dictionnairique.

Il établit des listes lexicales de deux types. L'une est intitulée Des mots et phrases qui sont en usage; c'est là que se situent les nombreuses références à Vaugelas, dans une approche qui s'inquiète de la 
synonymie. La seconde est un Traité des étymologies, qui explicite à la fois l'origine des mots considérés et la dérivation qui en provient éventuellement. Ce souci d'une analyse raisonnée du lexique est une originalité dans les grammaires du temps : on peut le relier à la fois aux travaux de Port-Royal, dont il était proche, et à l'élaboration de la première édition du Dictionnaire de l'Académie.

C'est surtout dans la seconde édition de la Méthode de Claude Irson, en 1660, qu'on trouve un développement maîtrisé des deux principales listes. Les acceptions des mots sont reliées de mieux en mieux à leur morphologie et à leurs emplois et la liste des étymologies s'enrichit de commentaires d'ordre scientifique et religieux, et raffine les champs lexicaux élaborés dans la première édition. Irson complète ses analyses par des considérations sémantiques liant la question de la polysémie à celle des genres.

Ainsi, pour Parallèle ou Période, qui peuvent être masculin ou féminin, Irson ne s'en tient pas à l'homonymie, il fait entrer en ligne de compte aussi bien la différence entre sens propre et sens figuré que les rôles des mots dans les syntagmes :

$$
\begin{aligned}
& \text { Parallèle étant pris au sens figuré est masculin, comme parallèle d'Alexandre et de César, c'est-à-dire la } \\
& \text { comparaison de l'un avec l'autre; mais il est féminin dans sa propre signification, comme lorsqu'on dit une } \\
& \text { parallèle, on sous-entend ligne dont toutes les parties sont également distantes de celles d'une autre ligne. }
\end{aligned}
$$

Dans la liste «des étymologies», Irson travaille dans un va-et-vient constant entre processus dérivationnel et évolution des sens, ce qui aboutit dans plusieurs cas à la constitution de micro-champs lexicaux qu'il explicite. Dans certains cas, l'origine dit le sens pour expliciter la dérivation :

Aiguille : vient d'aigu, d'où aiguillette, aiguillon, aiguillonner

Irson invoque l'héritage latin :

Aube signifie blanc, d'où on dit aube du jour, aubour, bois blanc

Et il montre parfois la complexité de l'histoire du lexique : un sens est origine d'une dérivation, et donc porte sur des dérivés, avec retour à une nouvelle origine donnant d'autres dérivés :

Brigand: insigne voleur d'où brigander, brigandage, brigantin, vaisseau de pirate dont les bords sont bas ; brigantine, habillement, brigand vient de brigue, comme qui dirait ceux d'une même brigue ou brigade: de là vient brigadier, chef d'une brigade.

Il va par là même vers la constitution de champs lexicaux :

Bègue, bégayer, bégaiement on remarquera que la voix de l'homme ayant plus d'inflexions est exprimée diversement : crier, bégayer servent proprement à exprimer le cri des enfants ; parler convient mieux à ceux qui raisonnent; radoter aux vieillards, babiller et caqueter aux grands causeurs ; cajoler aux courtisans, baragouiner aux étrangers qui écorchent la langue.

Quant à la liste des mots en usage, elle comprend également des élargissements importants en ce qui concerne la construction des verbes, ou la réception d'un mot dans l'usage contemporain, comme le montre le traitement de Féliciter, mot qu'il juge «bien reçu ». Et il explore une série de néologismes abstraits forgés directement sur le latin classique et en passe d'être acceptés : "conjoncture, intrépide, insidiateur, insidiatrice, sécurité et plusieurs autres ». On discerne là l'amorce d'une saisie organisée d'un lexique raisonné.

Certains mots figurent dans les deux listes et sont donc considérés à la fois dans leur usage et dans leur histoire. Ainsi, Bailler figure dans la Liste des mots en usage, dans la distinction entre bailler 1, donner, et bailler 2, ouvrir la bouche, et dans le Traité des étymologies, à propos de l'entrée Baillif: 
Et la suite de l'article nous éclaire sur des usages, passés et présents, de ce terme, dans les provinces et à l'étranger : Ce mot Baillif signifiait autrefois un Nourricier, parce qu'il porte l'enfant : d'où vient qu'on dit encore en
Languedoc une baille pour une nourrice. (...) Les vénitiens appellent aussi leur résident : Baille.

Ce grammairien, lexicologue avant la lettre, a donc su aller au-delà de son domaine strict pour travailler les mots de sa langue en choisissant autant ses points de départ que l'extension donnée à ses analyses et à ses commentaires.

\subsection{Vers le dictionnaire monolingue}

Pour conclure sur ces trois années (1656-1660) qui précèdent la publication de la Grammaire et de la Logique de Port-Royal, qui bouleverseront le paysage, on peut considérer que Chiflet est intéressant dans la mesure où ses observations sur le lexique ne sont pas atomisées comme dans les ouvrages de Remarqueurs mais se situent à l'intérieur d'une grammaire et sont reliées à la morphologie (parties du discours) et à la syntaxe, en particulier à la construction des verbes et aux syntagmes substantifs-adjectifs (les collocations). Mais il ne s'intéresse pas à l'exploration des différentes acceptions des mots et il ne travaille ni leur histoire ni les champs lexicaux dans lesquels ils sont pris.

Irson en revanche assure cette investigation, par exemple lorsqu'à l'entrée Roux, il traite non seulement Rouge, Rougeur et Rougeole mais aussi Rubrique, «table des livres ainsi nommée parce qu'on les écrivait en lettres rouges »; par là, et par delà l'hétérogénéité des formes, Irson explicite le lien qui réunit un champ lexical français à une origine latine et au surgissement d'un mot « savant».

Bref, on voit donc qu'à la fin du XVII siècle, deux voies se poursuivent : celle qui va vers les travaux de Girard, dans la recherche du mot juste et le raffinement de la distinction des sens d'un mot par rapport à ses synonymes, et celle de l'organisation du lexique en domaines morphosémantiques. Le Dictionnaire de l'Académie, dans son édition de 1694, réunira les deux, car même s'il n'est pas un dictionnaire de synonymes, le soin des définitions qu'on y trouve correspond à un travail soigneux sur les acceptions des termes, et le principe général de son organisation est celui d'une vision raisonnée du lexique français, comme le montre le texte même de sa préface :

\footnotetext{
Comme la langue française a des mots primitifs et des mots dérivés et composés, on a jugé qu'il serait agréable et instructif d disposer le Dictionnaire par racines, c'est-à-dire de ranger tous les mots dérivés et composés après les mots primitifs dont ils descendent, soit qu'ils viennent du latin ou de quelqu'autre langue. On s'est quelquefois dispensé de suivre cet ordre dans quelques autres mots qui, sortant d'une même souche latine, ont fait des braches assez diffférentes en Français pour être mis chacun à part ; et on s'en est souvent aussi dispensé dans quelques autre mots dont le primitif latin n'a point formé de primitif en français, ou a été aboli par l'usage, et dont par conséquent les dérivés et composés sont en quelque façon indépendants les uns des autres, comme les mots construire et destruire qui viennet du mot latin struere, qui n’a point passé en Français.
}

Dans cet arrangement de mots, on a observé de mettre les dérivés avant les composés, et de faire imprimer en gros caractères les mots primitifs comme les chefs de famille de tous ceux qui en dépendent, ce qui fait qu'on ne tombe guère sur un de ces mots primitifs qu'on ne soit tenté d'en lire toute la suite parce qu'on voit, s'il faut ainsi dire, l'histoire du mot, et qu'on en remarque la naissance et le progrès; et c'est ce qui rend cette lecture plus agréable que celle des autres dictionnaires qui n'ont pas l'ordre des racines.

Un peu avant la parution du DA, un ouvrage qui en est en quelque sorte complémentaire montre la réunion, à la fin du siècle, des travaux des grammairiens, des remarqueurs, et des lexicographes : c'est $\mathrm{Le}$ génie de la langue française de J. D'Aisy, publié en 1685. En effet, il contient un Abrégé de la grammaire française, puis des Remarques sur cette grammaire, les remarques formant l'essentiel de l'ouvrage. Or celles-ci sont tout à fait organisées; le lexique est considéré à travers un plan rigoureux et détaillé, qui va 
de la prononciation des mots à leur syntaxe, classe les termes en parties du discours, tient compte des dérivations et des constructions, et analyse les sens. En d'autres termes, ce livre d'un côté ramasse toutes les observations faites par Vaugelas, Ménage et Bouhours: il donne d'ailleurs à leurs remarques respectives un signe particulier (des icônes en marge). De l'autre, comme l'indique la préface, «il renferme sous un même article les remarques et les observations qui regardent une même difficulté, et qui sont néanmoins dispersées en plusieurs endroits des ouvrages de ces trois illustres auteurs ». Par là, l'on peut dire que le lexique est répandu à travers toutes les analyses de la grammaire, comme la grammaire est répandue dans tous les articles du DA.

D'aizy offre ainsi une mise en ordre dans un cadre général syntaxico-morpho-phonologique. Alors que l'Académie posera un noyau lexical de base, la « racine », et créera une véritable fabrique dérivationnelle, parfois violente dans sa synchronie et dans son refus de la distinction entre mots phonétiquements dérivés du latin et mots empruntés directement à cette langue, d'Aizy intègre son étude du lexique (troisième partie, chapitre 1, « De l'usage des mots ») dans une étude des instruments de dérivation : re, in+verbe, noms commençant par..., terminés en ..., ce qui lui permet de distinguer, morpho-phonétiquement, les emprunts et les dérivés.

Prenons le cas de la distinction des verbes «tout français »: ainsi remettre, de mettre, par rapport aux formes qui leur sont liées mais sont prises directement du latin comme rémission, et aux verbes qui commencent aussi par re- mais viennent directement du latin : réformer, réserver, réciter. D'Aizy appuie la distinction sur la prononciation du $e$ latin: "remettre et reprendre gardent toutjours la prononciation des verbes tout français, composés de re et des simples mettre et prendre. Mais on prononce toujours rémission et réprehension, des latins remissio et reprehensio." Dans le DA, au contraire, tout est unifié, aplani. Tout est devenu du français. Remission est à sa place alphabétique entre remise et remissionnaire et il n'y a pas de distinction entre les graphies-phonies de $r e$-puisqu'il n'y a jamais d'accent aigu sur le $e$ dans les mots de ce genre.

Il y a donc bien complémentarité entre ces deux ouvrages : d'un côté un cadre grammatical dans lequel s'insère un essai de systématisation du lexique français, de l'autre une approche raisonnée des familles de mots dans un système strict de l'arborescence des dérivations. Leurs regards n'ont pas le même objet: D'Aisy oppose ainsi reformer à réformer et à rétablir (re-établir). Alors que pour le DA, l'intérêt est que reformer vient de forme et que restablir, comme establir, vient de stable. (Le DA garde l'ancienne écriture en es- et non en é-). Pour reformer, étant donné l'absence d'accent, le DA donne un seul mot et distingue différents sens à l'intérieur de l'article.

Ces désirs d'organisation grammatico-lexicales obligent les deux ouvrages à proposer dans leurs dernières pages une liste de tous les mots traités dans l'ordre alphabétique strict.

\section{Pour conclure}

Le dictionnaire monolingue s'est donc fait par principes et non pour la pratique. D'autant que les querelles antérieures entre les auteurs de Remarques sont soit neutralisées (Académie), soit renvoyées aux protagonistes. Ainsi, à la fin du siècle, les divergences entre les observateurs de la langue sont neutralisées, l'ouvrage de Nicot de 1587, ce dictionnaire latin-françois « réduit à la forme et perfection des dictionnaires grecs et latins » a fait place à un dictionnaire de la « langue française » réduit à la forme et perfection d'une « langue commune », dans un outil linguistique anonymé. Un ouvrage d'envergure qui aura su «arrêter » la forme de la définition morphologique et de la définition d'usage (les collocations et domaines) contre les définitions et macrostructures des dictionnaires spécialisés et des dictionnaires de traduction.

On a ainsi, au début du siècle, un dictionnaire qui enferme une grammaire : celui de Nicot. Au milieu du siècle, des grammaires, comme celles de Chiflet ou celle d'Irson, qui renferment des listes de mots 
formant, dans le cas d'Irson, de véritables petits dictionnaires. Tout au long du siècle, il s'agit de montrer les règles, l'analogie du français, et en même temps, d'enregistrer les éléments lexicaux recevables et de les organiser, le tout en se dégageant de plus en plus du latin au fil des années. À la fin du siècle, D'Aisy explicite le "génie de la langue française » en instillant à travers tout un cadre grammatical des analyses serrées, et proprement linguistiques, du lexique de son temps, et le travail de l'Académie va aboutir à un dictionnaire monolingue qui systématisera de manière raisonnée le lexique français en se fondant sur une base grammaticale sous jacente. Autrement dit, la forme «dictionnaire de langue » ne nait pas d'une simplification du dictionnaire plurilingue. Cette forme est l'aboutissement de deux courants de recherche : celui des remarqueurs, et celui des grammairiens.

\section{Références bibliographiques}

Aizy (d'), J. (1685). Le génie de la langue française. Paris, chez Laurent d'Houry.

Arnauld, A. et Lancelot, C. (1660). Grammaire générale et raisonnée. Introd. de M. Foucault. Paris [rééd. Paris, Republications Paulet, 1969].

Auroux, S. (1997). «La réalité de l'hyperlangue ». Langages, n 127, p. 110-121.

Auroux, S. (1998). Le Langage, la raison et la norme. Paris, Presses Universitaires de France.

Bouhours, D. (1675). Remarques Nouvelles sur la langue française. [rééd. 1973, Genève, Slaktine Reprints].

Buridant, C. (1980). « Les binômes synonymiques. Esquisse d'une histoire des couples de synonymes du Moyen Âge $\mathrm{au} \mathrm{XVII}^{\mathrm{e}}$ siècle ». Synonymies, bulletin du centre d'analyse du discours, Université de Lille III.

Chiflet, L. (1659). Essay d'une parfaite grammaire de la langue française où le lecteur trouvera, en bel ordre, tout ce qui est de plus nécessaire, de plus curieux et de plus élégant, en la pureté, en l'orthographe et en la prononciation de cette langue. Anvers, chez Jacques Van Meurs.

Delesalle, S. (2007). «Le statut du lexique dans la Grammaire de Laurent Chifflet et dans les Méthodes de Claude Irson : 1656-1660 ». HEL, 28/2, p. 37-52 (voir infra)

Delesalle, S. et Mazière, F. (2002). «La liste dans le développement des grammaires ». HEL, 24-2, Paris.

Delesalle, S. et Mazière, F. (2003). « Meigret, la langue française et la tradition grammaticale». Dans : G. Defaux (dir. publ.), "Lyon et l'illustration de la langue française », Lyon, ENS éditions (coll. « Langages »).

Delesalle, S. et Mazière, F. (1998). «Raison, foi et usage. Les modes de la signification dans le dictionnaire de l'académie et la grammaire générale et raisonnée de Port-Royal », Sémiotique 14, p. 45-68.

Delesalle, S. et Mazière, F. (2007). « Grammaire générale et grammaire particulière : les méthodes de Claude Irson ». Actes du dixième colloque ICHOLS. Brésil. Août 2002.

Dictionnaire de l'Académie françoise (1694). 2 vols. Paris, Vve J.-B. Coignard et J.-B. Coignard.

Estienne, R. (1539-1549). Dictionnaire François-Latin, autrement dit les mots françois avec les manières d'user d'iceulx, tournés en latin. Paris, Robert Estienne.

Estienne, R. (1544). Les Mots françois selon l'ordre des lettres (françois-latin). Paris, Robert Estienne.

Furetiere, A. (1690) Dictionnaire Universel, contenant généralement tous les mots françois tant vieux que modernes, et les termes de toutes les sciences et des arts [...], La Haye/Rotterdam, Arnout \& Reinier Leers.

Girard (Abbé). (1718). La justesse de la Langue Française ou les différentes significations des mots qui passent pour synonymes. Paris, Vve d'Houry.

Girard (Abbé). (1736). Les Synonymes françois, leurs différentes significations et les choix qu'il faut faire pour parler avec justesse. Paris, $\mathrm{V}^{\mathrm{ve}} \mathrm{d}$ 'Houry.

Irson, C. (1656). Nouvelle Méthode pour apprendre facilement les principes et la pureté de la langue française. Paris, Pierre Baudouin [rééd. 1973, Genève, Slatkine reprints].

La Porte, M. de. (1571). Les épithètes. Paris, G. Buon.

Leclercq, O. (2006). "Le traitement du sens par les épithètes et les synonymes au XVI siècle: approche de la définition lexicale », HEL 28/2, p. 27-35.

Leclercq, O. (2008). « Autour d'un recueil de synonymes du XVI siècle : les premières traces de régulation du lexique français par le contexte ». Cahiers de Lexicologie. 
Leclercq, O. (2008). «Aux marges de la lexicographie : les recueils de proverbes aux XVI ${ }^{\mathrm{e}}$ et XVII ${ }^{\mathrm{e}}$ siècles ». Tenth International Conference on the History of the Language Sciences (ICHoLS X), University of Illinois at UrbanaChampaign, 1-5 sept. 2005.

Macé, J. (1651). Méthode universelle pour apprandre facilement les langues, pour parler et écrire nettement en françois, recueillie par le sieur Du Tertre. Seconde édition, 1651

Mazière, F. (1996). «Élaboration d'un dictionnaire de langue : Le dictionnaire de l’Académie (1694) et la préédition de 1687 ». Dans : S. Auroux et S. Delesalle (dir. publ.), Histoire et grammaire du sens. Paris, Armand Colin, p. 124-139.

Mazière, F. (2006). «Synonymie et définition. Ajustement des sens lexicaux en français ». Dans : Synonymie et 'differentiae': théories et méthodologies de l'époque classique à l'époque moderne. Actres du colloque international (Université de Messine, 6-8 octobre 2003). M. G. Adamo et P. Radici Colace (dir. publ.). Messine, Accademia Peloritana-Napoli, ESI.

Mazière, F. ( (2006). «Émergence de "la langue française"». Dans : E. Galazzi et C. Molinari (dir. publ.), Les français en émergence. Bern, Peter Lang.

Mazière F. (à paraître). «Les langues et le français dans la Méthode de Jean Macé (1651) ». Dans : T. Arnavieille et C. Camps (dir. publ.), Actes du colloque Discours et savoirs sur les langues anciennes et modernes, Béziers, 1921 oct. 2006. Paris, L'Harmattan.

Mazière, F et Auroux, S. 2007. "Introduction : Hyperlangues, modèles de grammatisation, réduction et autonomisation des langues ». Hyperlangues et fabriques de langues. HEL, 28/2, p. 7- 18.

Meigret, L. (1550). Le tretté de la grammere françoeze, fet par Louis Meigret Lionoes. Paris, Robert Estienne.

Ménage, G. (1675). Observations sur la langue françoise. Paris, C. Barbin.

Montméran, A. de. (1645). Synonimes et epithètes françoises. Paris, J. Le Bouc.

Nicot, J. (1606) Thresor de la langue françoyse, tant ancienne que moderne [...], Exact et très facile acheminement à la langue françoise [...].

Richelet, P. (1680). Dictionnaire françois contenant les mots et les choses, plusieurs nouvelles remarques sur la langue françoise [...]. Genève, Chez Jean Herman Widerhold.

Vaugelas, C. Favre de. (1647). Remarques sur la Langue Française. Paris, A. Courbé et V ${ }^{\mathrm{ve}}$ Camusat. [rééd. 1981, Champ libre].

Vivier, G. du (dit Vivre). (1569). Synonymes. C'est à dire plusieurs propos, propres tant en écrivant qu'en parlant, tirer quasi tous à un même sens, pour montrer la richesse de la Langue Français. Cologne, H. von Aich.

\footnotetext{
1 De façon générale, nous modernisons l'orthographe.

${ }^{2}$ Voir, en fin d'article, l'article Terre du Dictionnaire de l'Académie.
} 